\title{
Strong gamma frequency oscillations in the adolescent prefrontal cortex
}

\author{
Zhengyang Wang ${ }^{1}$, Balbir Singh², Xin Zhou ${ }^{2,3,4 *}$, Christos Constantinidis ${ }^{1,2,5}$ *
}

1. Neuroscience Program, Vanderbilt University, Nashville TN 37235, USA.

2. Department of Biomedical Engineering, Vanderbilt University, Nashville TN 37235, USA.

3. Department of Computer Science, Vanderbilt University, Nashville TN 37235, USA.

4. Data Science Institute, Vanderbilt University, Nashville TN 37235, USA.

5. Department of Ophthalmology and Visual Sciences, Vanderbilt University Medical Center, Nashville TN 37232, USA.

${ }^{*}$ Correspondence should be addressed to:

Christos Constantinidis (Christos.constantinidis.1@vanderbilt.edu) or

Xin Zhou (Maizie.zhou@vanderbilt.edu)

Abbreviated title: Strong gamma in the adolescent prefrontal cortex

Number of pages: 35

Number of figures: 8

Abstract length: 199 words

Introduction length: 502 words

Discussion length: 1495 words

Conflict of interest: The authors declare no conflicts of interest.

Acknowledgements: Research reported in this paper was supported by the National Institute of Mental Health of the National Institutes of Health under award numbers R01 MH117996 and R01 MH116675. We wish to thank Austin Lodish and Junda Zhu for technical and analytical help and André Bastos, Beatriz Luna, and David Blake for helpful comments on the manuscript. 


\section{Abstract}

31 Working memory ability continues to mature into adulthood in both humans and non-human

32 primates. At the single neuron level, adolescent development is characterized by increased

33 prefrontal firing rate in the delay period, but less is known about how coordinated activity

34 between neurons is altered. Local field potentials (LFP) provide a window into the computation

35 carried out by the local network. To address the effects of adolescent development on LFP

36 activity, three male rhesus monkeys were trained to perform an oculomotor delayed response

37 task and tested at both the adolescent and adult stage. Simultaneous single-unit and LFP

38 signals were recorded from areas $8 \mathrm{a}$ and 46 of the dorsolateral prefrontal cortex (dIPFC). In

39 both the cue and delay period, power relative to baseline increased in the gamma frequency

40 range $(32-128 \mathrm{~Hz})$. In the adult stage, high-firing neurons were also more likely to reside at

41 sites with strong gamma power increase from baseline. For both stages, the gamma power

42 increase in the delay was selective for sites with neuron encoding stimulus information in their

43 spiking. Gamma power and neuronal firing did not show stronger temporal correlations. Our

44 results establish gamma power decrease to be a feature of prefrontal cortical maturation. 


\section{Significance Statement}

46

47 Gamma-frequency oscillations in extracellular field recordings (such as LFP or EEG) are a

48 marker of normal interactions between excitatory and inhibitory neurons in neural circuits.

49 Abnormally low gamma power during working memory is seen in conditions such as

50 schizophrenia. We sought to examine whether the immature prefrontal cortex similarly exhibits

51 lower power in the gamma frequency range during working memory, in a non-human primate

52 model of adolescence. Contrary to this expectation, the adolescent PFC exhibited stronger

53 gamma power during the maintenance of working memory. Our findings reveal an unknown

54 developmental maturation trajectory of gamma band oscillations and raise the possibility that

55 schizophrenia represent an excessive state of prefrontal maturation. 


\section{Introduction}

Human executive functions including working memory continue to mature after the onset of puberty (Fry and Hale, 2000; Gathercole et al., 2004; Davidson et al., 2006; Ullman et al., 2014). This pattern of maturation parallels continued structural changes in the prefrontal cortex:

61 early childhood excitatory synaptogenesis is followed by synaptic pruning in adolescence

62 (Bourgeois et al., 1994; Anderson et al., 1995; Huttenlocher and Dabholkar, 1997), along with

63 decreasing cortical thickness and gray matter volume (Giedd and Rapoport, 2010). In tasks

64 requiring working memory, human imaging studies have also reported distinct changes in

65 prefrontal activity patterns in humans between childhood and adulthood (Luna et al., 2001;

66 Bunge et al., 2002; Klingberg et al., 2002; Kwon et al., 2002; Olesen et al., 2003; Burgund et al.,

67 2006; Olesen et al., 2007). Other markers of neuronal activity, including the distribution of power

68 across different frequency bands of the EEG also changes markedly between the time of

69 adolescence and adulthood (Uhlhaas et al., 2009). Gamma-band oscillations are thought to be

70 driven primarily by excitatory-inhibitory neuronal loops (Buzsaki and Wang, 2012), thus

71 suggesting that strength of gamma oscillations can serve as a marker of synaptic maturation

72 (Uhlhaas et al., 2010). Schizophrenia, a neurodevelopmental disorder with a typical early

73 adulthood onset are characterized by impaired working memory performance (Goldman-Rakic,

74 1994), abnormal trajectory of prefrontal interneuron maturation (Dienel and Lewis, 2019) as well

75 as decreased power of gamma oscillations (Woo et al., 2010; Uhlhaas and Singer, 2013).

Non-human primates exhibit a strikingly similar pattern of cognitive development and

77 prefrontal maturation (Constantinidis and Luna, 2019) and allow for more direct insights in the

78 nature of neural changes that mediate these phenomena. Single-neuron recordings obtained in

79 non-human primates at different developmental stages have thus revealed increased firing rate

80 specifically during the intervals of memory maintenance (Zhou et al., 2013; Zhou et al., 2014; 
81 Zhou et al., 2016b). Continued maturation of inhibitory connections has also been directly

82 documented in the adolescent prefrontal cortex (Gonzalez-Burgos et al., 2015), as have been

83 changes in the effective, intrinsic connectivity between prefrontal neurons between adolescence

84 and adulthood (Zhou et al., 2014). Analysis of neuronal rhythmicity in the adolescent primate

85 prefrontal cortex has not been reported until now. Local filed potentials (LFP), a signal

86 representative of the summation of postsynaptic activity in a small cortical volume (Kajikawa

87 and Schroeder, 2011), provide a mesoscopic measure to link these empirical results. As in

88 human EEG studies, working memory maintenance is generally characterized by elevated

89 gamma-frequency power in the local field potential (Pesaran et al., 2002; Howard et al., 2003;

90 Jensen et al., 2007; Honkanen et al., 2015; Kornblith et al., 2016).

91 We were thus motivated to determine whether prefrontal cortical maturation between

92 adolescence and adulthood is characterized by increased gamma-band rhythmicity in the local

93 field potential. We analyzed LFP recordings obtained from the same subjects as the transitioned

94 from adolescence to adulthood (Zhou et al., 2016b; Zhou et al., 2016c). Unexpectedly, we found

95 robust gamma band oscillations in the adolescent prefrontal cortex that declined rather than

96 increased during adulthood. 


\section{Methods}

\section{Subjects and data collection}

100 Neurophysiological data used for analysis was collected from male rhesus monkeys performing

101 a visual working memory task and reported in detail previously (Zhou et al., 2013; Zhou et al.,

102 2014; Zhou et al., 2016a; Zhou et al., 2016c). All surgical and animal use procedures were

103 reviewed and approved by the Wake Forest University Institutional Animal Care and Use

104 Committee, in accordance with the U.S. Public Health Service Policy on humane care and use

105 of laboratory animals and the National Research Council's Guide for the care and use of

106 laboratory animals. Quarterly morphometric and hormonal measures were obtained to

107 determine each animal's onset of puberty as well as maturity.

108 The monkeys were trained to perform the ODR task (Fig. 1). This task required the subject to

109 remember the location of a $1^{\circ}$ white square stimulus presented for $0.5 \mathrm{~s}$ following a $1 \mathrm{~s}$ fixation

110 period. The stimulus could appear at one of eight locations arranged on a circle of $10^{\circ}$

111 eccentricity. After a 1.5 s delay period, the fixation point was extinguished, prompting the

112 subject to saccade to the remembered location within a $0.6 \mathrm{~s}$ time window to receive a liquid

113 reward. The saccade endpoint had to deviate no more than $5-6^{\circ}$ from the center of the stimulus.

114 Correct trials were included for subsequent analysis.

115 Neural recordings were collected from areas $8 \mathrm{a}$ and 46 of the dorsolateral prefrontal cortex.

116 Epoxylite-coated Tungsten electrodes with a diameter of $250 \mu \mathrm{m}$ and an impedance of $4 \mathrm{M} \Omega$ at

$1171 \mathrm{KHz}$ (FHC Bowdoin, ME) were acutely advanced into the brain, and a reference electrode was

118 attached to the metal recording chamber. Spike waveforms were amplified, band-pass filtered

119 between 0.5 and $8 \mathrm{kHz}$, and sampled at $40 \mathrm{kHz}$, while unipolar LFP traces were sampled

120 continuously at $500 \mathrm{~Hz}$. Both signals were digitized and stored through a modular data

121 acquisition system (APM system, FHC, Bowdoin, ME). 


\section{LFP signal processing}

123 LFP recordings were processed using the FieldTrip (https://www.fieldtriptoolbox.org/) and

124 Chronux (http://chronux.org/) toolboxes as well as custom MATLAB code in MATLAB 2018b

125 (MathWorks). LFP signals of individual trials first underwent artifact rejection. Single-trial LFP

126 traces were zero-meaned and then band-pass filtered between 1 and $200 \mathrm{~Hz}$ with a zero-phase

$1275^{\text {th }}$ order Butterworth filter $\left(10^{\text {th }}\right.$ order in effect) and then notch-filtered at $60 \mathrm{~Hz}$ with a bandwidth

128 of $0.2 \mathrm{~Hz}$ using the ft_preproc_dftfilter function. The signals were then standardized by their

129 standard deviation as estimated by the median absolute deviation. The spectrogram of single

130 traces between 2 and $128 \mathrm{~Hz}$ were computed by the mtspecgramc function with 6 tapers of 500

131 ms time windows. LFP power in the alpha, beta, gamma and high-gamma bands were defined

132 to be the sum of power in the spectrogram between the frequencies of $8-16 \mathrm{~Hz}, 16-32 \mathrm{~Hz}$,

$13332-64 \mathrm{HZ}$ and $64-128 \mathrm{~Hz}$ respectively. We used the last $500 \mathrm{~ms}$ of the fixation period as the

134 baseline period. Relative band power was calculated by dividing the sum of power within each

135 band at each time point by the average of the sum in the baseline period. The values in the

136 relative spectrograms (Fig. 2) were calculated separately at each frequency (at a 2-Hz

137 resolution). To identify recording sites where LFP power was modulated in a specific frequency

138 band, a two-tailed paired-sample t-test was carried out on the band power from all trials at each

139 recording site averaged during the delay period. Sites with $p$ values below 0.05 was defined to

140 be modulated in the corresponding frequency band. To find spatially selective LFP recording

141 sites, 1-way ANOVA test was carried out on the band power of each recording site averaged

142 during the delay period in response to different stimulus location. LFP recording sites with $p$

143 values below 0.05 were defined to be selective for stimulus location in the corresponding power

144 band. 


\section{Spike processing}

146 Recorded spike waveforms were sorted into separate units using an automated cluster analysis

147 method based on the KlustaKwik algorithm. Trial-averaged PSTH was computed by convolving

148 the spiking events with a 50-ms boxcar kernel at 20-ms steps apart. The evoked PSTH was

149 calculated by subtracting the average firing rate in the 1-second fixation period. Single-trial

150 PSTH for computing moment-by-moment percentage of explained variance (PEV) used a 250-

151 ms box kernel instead. Neurons were identified to be responsive to the task, if their activity

152 increased significantly during any task epoch relative to the baseline fixation period, evaluated

153 at the 0.05 significance level, as we have described previously (Zhou et al., 2016c). To identify

154 neurons selective for spatial location during the delay period of the task, a 1-way ANOVA test

155 was carried out on the firing rates of each neuron averaged during the entire delay period.

156 Selective neurons were defined to be the ones with $p$ values below 0.05 . The bias-corrected

157 PEV $\omega^{2}$ was computed in equation (1) at steps of $20 \mathrm{~ms}$ on the single-trial firing rate of each

158 neuron, where MSE is the mean squared error within stimulus locations, df the degree of

159 freedom, $S_{\text {Between }}$ the sum of squares between stimulus locations and $S_{\text {total }}$ the total variance.

$$
\omega^{2}=\frac{\mathrm{ss}_{\text {Between }}-\mathrm{df} \times \mathrm{MSE}}{\mathrm{ss}_{\text {Total }}+\mathrm{MSE}}
$$

Cluster-based permutation test

162 A permutation test with 10,000 iterations was carried out to compare the relative spectrogram

163 between the adolescent and the adult using the FieldTrip function ft_freqstatistics. two-tailed

164 independent-sample t-tests were first run for each time-frequency combination to generate

165 uncorrected $p$ values before connected regions of all positive or negative t statistics with $p$

166 values smaller than 0.05 were identified (clusters). The sum of all t statistics within each cluster

167 were calculated. The labels for developmental stage were then randomly shuffled 10,000 times

168 where each time the largest cluster sum of $t$ values was recorded. The original cluster sum of $t$ 
values was then compared against the bootstrapped shuffled distribution to generate a one-

170 tailed $p$ value.

171

172 Correlation analysis

173 The neuron-by-neuron Spearman's correlation between neuronal firing rate/PEV and LFP power

174 was computed by first averaging the signals within each neuron or recording site and within the

175 delay period and then matching the signals that were recorded from the same electrode in each

176 session. The correlation coefficients were compared across the adolescent and the adult using

177 Fisher's Z transform.

Temporal correlation was calculated for the time-series signals in the delay period of

179 each trial. The spiking events were convolved with a 50-ms boxcar kernel. For each neuron, the

180 trials for each stimulus location were concatenated and the correlation was computed between

181 the convolved spiking and the LFP power changes from baseline at each frequency. Only

182 results from the stimulus location that resulted in the strongest evoked delay period firing rate

183 for each neuron were further considered. When examined on a frequency-by-frequency basis,

184 all $p$ values were controlled for using the Bonferroni-Holm procedure (see the Statistics section

185 for details).

\section{Statistics}

188 All statistical tests were conducted using MATLAB 2018b. An alpha level of 0.05 was adopted

189 for all tests. The family-wise error rate (FWER) was controlled for using the Bonferroni

190 correction when multiple comparisons were conducted in multiple canonical frequency bands.

191 For $n$ comparisons, the Bonferroni correction sets the critical $p$ value to $\alpha / n$. Alternatively, the

192 Bonferroni-Holm procedure was carried out when multiple comparisons were conducted in a 
193 frequency-by-frequency fashion. Briefly, the Bonferroni-Holm procedure sorts all $p$ values from

194 lowest to highest, then compares in order from the first to the $k$ th $\mathrm{p}$ value to a critical level of

$195 \alpha /(n+1-k)$. A comparison is considered significant if the current $\mathrm{p}$ value is smaller than the

196 critical level, otherwise the current and all following comparisons are deemed not significant,

197 and the procedure stops. 


\section{Results}

199 Data were analyzed from three male Rhesus monkeys (Macaca mulatta), trained to perform the 200 oculomotor delayed response (ODR) task (Fig. 1B). The task tested the animals' visual working 201 memory ability by requiring the subject, after a delay of 1.5 second, to make a saccade to a 202 remembered stimulus location that was presented for 0.5 seconds. The animals were tested at 203 two stages of development: the 'adolescent' stage after puberty onset (approximately age 4.5

204 years) and the 'adult' stage once development had completed (approximately age 6.5 years).

205 Young monkeys generally achieved a lower performance in the task than adult ones (Fig. 1C, $20688 \%$ vs. 98\% correct performance for young and adult, respectively, not considering aborted 207 trials), as we have reported previously (Zhou et al., 2016c). During performance of the task, 208 spiking and LFP activity was recorded from area 8a and 46 (Fig. 1A). We analyzed LFP activity 209 from electrodes where single unit activity was also identified. The resulting dataset consisted of 210 signals from 298 neurons and 130 LFP sites in the adolescent stage along with 392 neurons 211 and 164 LFP sites in the adult stage.

Adolescent dIPFC shows a stronger pattern of LFP power modulation in the ODR task

214 We calculated LFP spectral power at each epoch of the ODR task, relative to baseline. For each 215 recording session from one electrode, the baseline period for LFP analysis was defined to be 216 the last 500 milliseconds of the fixation period in order to avoid including the transient power 217 changes following fixation onset. The relative power of the recording was then calculated as the 218 spectrogram change from the baseline average at each respective frequency. Results from 219 different sessions and electrodes were averaged together for the adolescent (Fig. 2A) and the 220 adult stage (Fig. 2B) respectively. In order to describe in detail its temporal evolution throughout

221 the different epochs of the task, LFP power modulation was evaluated in four different frequency 222 bands: alpha $(8-16 \mathrm{~Hz})$, beta $(16-32 \mathrm{~Hz})$, gamma $(32-64 \mathrm{~Hz})$ and high-gamma $(64-128$ 
$223 \mathrm{~Hz}$ ). The resulting relative power spectrogram showed similar patterns of modulations in each

224 task epoch at the adolescent and the adult stage. Specifically, gamma (Fig. 2E) and high-

225 gamma (Fig. 2F) power were elevated relative to baseline during both the cue presentation and

226 the delay period. Beta power (Fig. 2D) generally showed modulations in the opposite direction

227 as gamma, showing decreases in both the cue and delay periods. Alpha band power (Fig. 2C)

228 had an initial increase during cue presentation and then quickly dropped below baseline in the

229 delay period.

Contrary to our initial hypothesis, gamma-band and high-gamma band LFP power was more strongly elevated during the delay interval of the task in the adolescent than the adult stage (Fig. 2E-F). Averaged across the entire duration of the delay period, the difference was highly significant for the gamma (two-tailed t-test $t(292)=5.09, p=6.4 E-7$ ) and high-gamma range (two-tailed t-test $t(292)=4.86, p=1.9 E-6)$. This difference was specific for the gamma and high-gamma frequency ranges. No significant difference was detected for the alpha or beta power (two-tailed t-test $\mathrm{t}(292)=-0.13$ and -0.68 respectively; $\mathrm{p}=0.89$ and 0.50 respectively). In addition, as previously reported, adult dIPFC neurons featured higher firing rates, both in terms

238 of absolute firing rate and as an increase over the baseline. This was true for the subset of 239 neurons (Fig. 4A, C) recorded in electrode penetrations where these LFPs were recorded. As a 240 result, the difference in gamma and high-gamma power between the two stages cannot be 241 accounted for by systematic differences in firing rate and in fact moved in the opposite direction 242 than would be expected based on firing rate alone. 
248 epochs. One other marginally significant $(p=0.0457)$ cluster was found suggesting stronger 249 negative modulation for the adolescent below $30 \mathrm{~Hz}$ in an epoch immediately following cue 250 presentation.

In order to rule out potential biased sampling of recording sites with heterogeneous LFP

252 power properties, we defined a site to be LFP power-modulated if its delay period power in a certain frequency band differed significantly relative to baseline (evaluated with a paired t-test,

254 at the $\alpha=0.05$ significance level). Most adolescent and adult sites were gamma power modulated, but more so adolescent (adolescent: 104/130; adult: 97/164, Fisher's exact test, $p=$

257 (adolescent: 117/130; adult: 122/164, Fisher's exact test, $p=8.1 \mathrm{E}-4$ ). We repeated the comparison of gamma power between the young and adult stages, including in the analysis exclusively the power-modulated sites. A higher power in the adolescent than in the adult stage was still present in the gamma (Fig. 3A, two-tailed t-test $t(199)=3.07, p=2.5 E-3$ ) and high-

261 gamma frequency band (Fig. 3B, two-tailed t-test $t(237)=3.31, p=1.1 E-3)$. The observed 262 differences in gamma and high-gamma delay power were thus unlikely due to unequal sampling 263 of modulated vs. non-modulated sites.

265 (Pesaran et al., 2002). We therefore wished to test whether the higher adolescent gamma 266 power was only evident in sites selective for the stimulus location or in sites that were not 267 modulated by the stimulus. We defined spatially-selective LFP sites as those in which delay 268 period power differed significantly depending on the location of the stimulus (evaluated with 1269 way ANOVA at the $\alpha=0.05$ significance level). Similar percentages of adolescent and adult LFP 270 sites exhibited spatially-selective gamma power (adolescent: 31/130; adult: 28/164) and high271 gamma (adolescent: 41/130; adult: 52/164). In the spatially-selective sites, gamma and high272 gamma delay-period LFP power was higher overall in the adolescent than the adult stage, but 
273 the difference did not reach significance for the gamma range (two-tailed t-test $t(57)=0.78, p=$

2740.44 ); it only did for high-gamma power (two-tailed t-test $t(91)=3.55, p=6.1 \mathrm{E}-4)$. Among non-

275 spatially-selective sites, delay-period LFP power was significantly higher in the adolescent stage

276 for both the gamma (two-tailed t-test $\mathrm{t}(233)=5.31, \mathrm{p}=2.6 \mathrm{E}-7$ ) and high-gamma frequency band

277 (two-tailed t-test $\mathrm{t}(199)=3.89, \mathrm{p}=1.4 \mathrm{E}-4)$.

\section{Adolescent neurons' delay period firing correlates less with gamma}

280 Gamma oscillations have been suggested to reflect the underlying synchronization of local

281 networks giving rise to delay period activity as well as differential encoding of stimulus

282 information in working memory (Roux and Uhlhaas, 2014). Given that we have previously

283 reported higher firing rate in the delay period during the adult stage (Zhou et al., 2016c), it

284 seemed curious that delay period gamma power and firing rate saw changes in opposite

285 directions during adolescent development. Therefore, we tested for potential changes in

286 correlation at the neuronal population level between gamma power modulation and neuronal

287 spiking. A neuron's preferred stimulus location was defined as the one that evoked the highest

288 average firing rate in the cue and delay periods combined. The correlation between neuronal

289 firing and LFP power was overall higher in the adult and emerged amongst a broader band of

290 frequencies (Fig. 5A). In the adult stage, the neurons' evoked delay period firing rates to the

291 preferred stimulus location weakly but significantly correlated with gamma (Spearman's rho =

292 0.326, $p=4.0 \mathrm{E}-11$ ) and high-gamma power modulations in LFP signals recorded at the

293 corresponding sites (Spearman's rho $=0.513, p=1.2 \mathrm{E}-27$ ). The strength of such correlations

294 was weaker in the adolescent stage for the gamma (Spearman's rho $=0.170, p=0.003$ ) and

295 high-gamma (Spearman's rho $=0.360, p=1.6 \mathrm{E}-10$ ) bands respectively. The adult correlation

296 between delay period firing rate and high-gamma band LFP was significantly higher than the 
297 young one (Fisher's Z-transform, gamma: $p=0.031>0.05 / 2$; high-gamma: $p=0.014<0.05 / 2$ 298 under Bonferroni correction).

In order to discount the potential contribution of firing rate differences (adolescent: $5.79 \pm$ 7.04 vs. adult: $7.44 \pm 8.28$ spikes per second) to gamma power through spectral leakage, we assembled an evoked firing rate-matched subset of data by removing the top $5.6 \%(22 / 392)$ of adult neurons and bottom 5.0\% (15/298) of adolescent neurons in terms of evoked firing rate in the delay period to the preferred stimulus location. These remaining adolescent neurons had an average evoked firing rate of $6.16 \pm 7.04$ spikes per second, matching the $6.01 \pm 5.49$ spikes per second of the remaining adult neurons (two-tailed t-test, $t(651)=0.32, p=0.75$ ). The reduced dataset's delay evoked firing showed a very similar pattern of correlations (Fig. 5B) as the full dataset between gamma (adolescent: Spearman's rho $=0.160, p=0.007$; adult: Spearman's rho $=0.335, p=4.0 \mathrm{E}-11$ ) and high-gamma power modulations (adolescent: Spearman's rho $=0.339, p=4.9 E-9$; adult: Spearman's rho $=0.490, p=1.0 \mathrm{E}-23)$. After matching for evoked neuronal firing rates, the adult stage still showed significantly stronger

311 correlations than the adolescent one (Fisher's Z-transform, gamma: $p=0.018<0.05 / 2$; high-

312 gamma: $p=0.021<0.05 / 2$ under Bonferroni correction). Furthermore, these differences in

313 correlation cannot be explained by the mismatch of the two stages' gamma power either, for the

314 adolescents' higher gamma power average should lead to higher correlation coefficients if the

315 underlying true distribution of correlations were the same between the two stages. The results of 316 this analysis suggest that network activity may be constraining unit responses more in the adult 317 than the adolescent stage.

320 In analogy to spatially-selective LFP sites, we identified spatially-selective single neurons if their 321 average evoked firing rates in the delay period were significantly different across stimulus 
322 locations and 'non-selective' otherwise (see Methods). This resulted in 39\% (116 out of 298) of 323 adolescent neurons and 40\% (157 out of 392) of adult neurons being categorized to be

324 spatially-selective. The ratio of selective to non-selective neurons did not differ significantly 325 between the 2 stages (Fisher's exact test, $p=0.81$ ). Amongst the selective neurons, those in 326 the adult had higher evoked firing rate in the delay period to their preferred stimulus locations 327 (Fig. 4B; two-tailed t-test, $\mathrm{t}(271)=2.98, \mathrm{p}=0.003$ ) as well as higher PEV (Fig. 4D; two-tailed t328 test, $\mathrm{t}(271)=2.71, \mathrm{p}=0.007)$. We used a 2-way ANOVA to compare delay-period LFP power in 329 sites where selective and non-selective neurons were recorded. LFP delay period gamma (Fig. 330 6C) and high-gamma (Fig. 6D) power was higher at sites where selective neurons were 331 recorded, evidenced by a significant main effect of selective-neuron site (gamma: $F(1,290)=$ 332 24.78, $p=1.1 \mathrm{E}-6$; high-gamma $F(1,290)=25.04, p=9.8 E-7)$. There was also a significant main 333 effect of developmental stage (gamma: $F(1,290)=24.85, p=1.1 E-6$; high-gamma: $F(1,290)=$ $33422.42, p=3.4 \mathrm{E}-6)$, but no interaction between the selective-neuron site and developmental stage (gamma: $F(1,290)=3.30, p=0.07$; high-gamma: $F(1,290)=0.16, p=0.69)$. gamma-modulation. Indeed, selective neurons were more likely to be found at gammamodulated recording sites compared to non-selective neurons in both the adolescent (selective neurons: $95.7 \%, \mathrm{~N}=116$; non-selective neurons: $85.7 \%, \mathrm{~N}=182$; Fisher's exact test, $\mathrm{p}=$ 0.006 ) and the adult (selective neurons: $74.5 \%, \mathrm{~N}=157$; non-selective neurons: $58.3 \%, \mathrm{~N}=$

$341235 ;$ Fisher's exact test, $p=0.001$ ) stage. PEV of neuronal discharges (Fig. 5C) revealed that for both the adolescent and adult neurons,

344 there was significant correlation between neuronal information and LFP power in the gamma and high-gamma frequency range (Bonferroni-Holm-corrected type I error rate $<0.05$ ). Similar 
347 between the adolescent and the adult at any frequencies (Fisher's Z transform, Bonferroni-

348 Holm-corrected), indicating the association between gamma power and neuronal information

349 encoding remained highly similar in both developmental stages. For the reduced dataset with

350 matched firing rates for the adolescent and adult neurons, the distribution of correlations was

351 still highly consistent between the two stages (Fig. 5D).

No strong temporal correlation between LFP power modulations and neuronal spiking

354 LFP oscillations in the dIPFC have been previously suggested to control neuronal spiking and

355 thus information encoding in a moment-by-moment fashion through their transient temporal

356 dynamics (Lundqvist et al., 2016; Lundqvist et al., 2018). In order to test at the single-trial level,

357 how much the delay period moment-by-moment change in gamma and high gamma power

358 reflected neuronal spiking, we extracted, for each neuron's preferred stimulus trials, their firing

359 rate (convolved with a 50-ms boxcar kernel) and band power traces. On single trials, gamma

360 power often exhibited short-living peaks surrounded by decreases in power. When single trials

361 from all recording sites were pooled according to developmental stage, gamma power exhibited

362 peak time points throughout the delay period (Fig. 7A-B). Both the adolescent and adult gamma

363 power peak times followed a similar distribution (two-sample Kolmogorov-Smirnov test $D(6185)$

$364=0.03, p=0.131)$. The trial-by-trial spiking of the matching neurons on the other hand, showed

365 no evidence of activity clustered at these gamma peaks and thus bore little temporal structure

366 similarity (Fig. 7C-D). This was to be expected according to previous accounts of prefrontal

367 neurons exhibiting higher temporal irregularity in the mnemonic delay period with the majority of

368 whose behavior mimicking a Poisson process (Compte et al., 2003). Consequently, the gamma

369 power showed little to no correlation on average with those of neuronal firing at the

370 corresponding recording sites in either the adolescent (Spearman's correlation, mean rho =

$3710.045, \mathrm{~N}=2792$ ) or adult stage (Spearman's correlation, mean rho $=0.027, \mathrm{~N}=3393$ ). 
372 Shuffling the trial pairings between the gamma power and firing rate for 100,000 iterations within

373 in each age group (adolescent $95 \%$ confidence interval: -0.004 to 0.011 ; adult $95 \%$ confidence

374 interval: -0.006 to 0.011 ) showed that the observed mean correlations were significantly higher

375 than chance.

376 Similar analysis was carried out on the temporal profile of high-gamma power (Fig. 7E-

$377 \mathrm{H}$ ). Interesting, the distribution of peaking time differed significant between the two stages (two-

378 sample Kolmogorov-Smirnov test $\mathrm{D}(6185)=0.08, \mathrm{p}=1 \mathrm{E}-9)$, with the adolescent high-gamma

379 power peaking earlier overall in the mnemonic delay. Furthermore, there was a weak temporal

380 correlation between high-gamma power and neuronal firing at both the adolescent (Spearman's

381 correlation, mean rho $=0.111, \mathrm{~N}=2792$ ) and adult stage (Spearman's correlation, mean rho =

$3820.127, \mathrm{~N}=3393)$. Such correlations were abolished once the trial pairings were shuffled

$383100,000$ times for either the adolescent (95\% confidence interval: -0.007 to 0.010$)$ or adult trials

384 ((95\% confidence interval: 0 to 0.015$)$.

While the correlation between high-gamma power and firing rate is a common

observation (Ray and Maunsell, 2011), the absolute level of correlation observed in this dataset

387 was rather low. Furthermore, spectral leakage from spiking might be responsible for part of this

388 correlation, which would further reduce the magnitude of any true correlations. A frequency-by-

389 frequency inspection of temporal correlations between neuronal firing and LFP powers showed

390 that the correlation coefficient increased with frequencies for both the adolescent and the adult,

391 further suggesting the contribution of spectral leakage from spikes at higher frequencies (Fig. 8).

392 The positive correlations became significantly different from 0 starting at $36 \mathrm{~Hz}$ and $42 \mathrm{~Hz}$ for

393 the adolescent and the adult respectively. It is also worth noting that, opposite to the

394 developmental effect on neuron-by-neuron correlations, the temporal correlation between LFP

395 power and spiking was higher for the adolescent than the adult, mostly in the high-gamma

396 range. This is further evidence that the stronger association between gamma power and high- 
397 firing neurons in the adult was not due to spectral leakage into the LFP directly from spiking

398 activity. In summary, gamma power and neuronal spiking did not follow closely the same

399 temporal profile from trial to trial. The relationship between the temporal dynamics of neuronal

400 stimulus encoding and moment-by-moment LFP power modulation in the delay period showed a

401 wide distribution and were not governed by a single rule across neurons. The adolescent and

402 adult stages only seemed to differ in the distribution of the peak timing of high-gamma power

403 from trial to trial. 


\section{Discussion}

406 Here, we analyzed the spiking and LFP signals from the dIPFC of monkeys at the adolescent

407 and adult stage respectively, while they performed a visual working memory task. Previous

408 studies have documented that the adult dIPFC is characterized by increased delay period firing

409 rate (Zhou et al., 2016c), yet we now saw an overall decrease of LFP gamma-band power in the

410 delay period of the task in adult monkeys. A similar change was present for high-gamma power,

411 while alpha and beta band power remained relatively stable during adolescent development.

412 This gamma-power decrease was evident when studying exclusively recordings from sites that

413 exhibited significant gamma power increase in the delay period, suggesting that the findings

414 were not due to any unbalanced sampling of potentially heterogeneous populations of local

415 circuits in the dIPFC. In both the adolescent and adult dIPFC, gamma power was higher at sites

416 where neuronal spiking contained significant stimulus information in the delay period.

417 Interestingly, this separation was largely not interacting with between-stage differences (Fig. 4,

418 5). These findings, along with the observation that the two stages had almost identical

419 percentages of selective neurons, indicates that peripubertal development of the primate dIPFC

420 does not abolish the already established computational principles in the local circuitry: stronger

421 gamma oscillations would emerge in a local network of neurons actively maintaining task-

422 relevant stimulus information. Rather, the adult PFC differed from the adolescent by having

423 high-firing, information-encoding neurons more heavily distributed at sites with stronger gamma

424 power. This finding, along with the observation that the increase in mature delay period firing

425 rate was biased for selective neurons (Fig. 4) suggests a potential refinement strategy in

426 adulthood: compared to the immature dIPFC, the adult prefrontal cortex more efficiently recruits

427 a task-relevant subset of local networks, engaging them in reverberatory dynamics that are

428 more localized and less diffuse so as to result in lower magnitude of gamma power in population 
measures such as the LFP. The universal differences seen across all populations of the recording sites are likely to be attributed to overall changes in synaptic properties including

431 synaptogenesis and receptor expression (Lewis et al., 2004; Hoftman and Lewis, 2011;

432 Gonzalez-Burgos et al., 2015), leading to a shift in the excitation-inhibition balance and 433 consequently the level of neural synchronization (Compte et al., 2000).

436 The temporal relationship between neuronal spiking and gamma power is complex. During

437 stimulus presentation, the highly correlated bottom-up inputs can serve to synchronize

438 population neuronal spiking and phases of synchronized excitation by pyramidal neurons

439 followed by inhibition by interneurons can thus produce oscillations in the field potentials (Fries,

440 2009). However, LFP gamma power can also emerge in the delay period, when no sensory

441 input is present, and the postsynaptic potentials are dominated by the local recurrent

442 connections (Pesaran et al., 2002). As modelling findings have suggested, high-frequency

443 oscillations readily emerge in a strongly recurrent network in which single neuron firing is highly

444 irregular, without exhibiting synchronization amongst a sparsely sampled subset of neurons

445 (Wang, 2010). Previous recordings have described dIPFC firing during the delay to be close to

446 Poisson-like (Compte et al., 2003) and Fano factor values above 1 for the adolescent and adult

447 neurons alike (Zhou et al., 2016c). The spatial scope of LFP signals dictates that they reflect the

448 summation of activity from a wide range of neurons (Kajikawa and Schroeder, 2011). Given the

449 highly irregular nature of dIPFC neuronal firing, it is reasonable that LFP would not be highly

450 predictive of activity recorded from a small subset of single neurons from the local population of

451 unsynchronized firing and various stimulus tuning. This was also supported by the fact that a

452 smaller percentage of LFP sites showed significant spatial tuning compared to that of neuronal

453 spiking. 
Local-circuit differences have been described between adolescent and adult monkeys that could explain changes in persistent discharges and gamma-band LFP oscillations between

456 developmental stages (Li et al., 2020). Zero-lag spiking synchronization based on cross-

457 correlation analysis of nearby neurons (recorded at distances between $0.5-1 \mathrm{~mm}$ from each

458 other) is markedly lower in adolescent than in adult monkeys. This difference is primarily the

459 effect of changes in inhibitory interactions, the net efficacy of which declines in adulthood (Zhou

460 et al., 2014). Anatomical evidence, in turn, implicates decreases in the connectivity strength of

461 pyramidal neurons onto interneurons, which lessens the net output of inhibitory connections as

462 the prefrontal cortex matures (Gonzalez-Burgos et al., 2015). This pruning of synaptic

463 interactions implies less synaptic drive overall, which could explain our current finding of

464 decreased rather than increased gamma-band power in the LFP.

466 Cognitive processes related to gamma power maturation

467 Results from human EEG/MEG literature have indicated systematic changes in rhythmicity

468 between developmental stages, generally suggestive of higher gamma power in adulthood.

469 However, closer examination of these studies reveals that existence and direction of change in

470 gamma power is not universal, but dependent on the specific brain region, task epoch and

471 nature of task performed. Many developmental EEG studies utilized resting state EEG that

472 reflects the network at a different state compared to when it is actively engaged in a working

473 memory task, or relied on passive sensory tasks, which revealed the greatest effects in lower

474 cortical areas, including stronger adult $40-\mathrm{Hz}$ auditory steady-state response dominantly

475 generated in the auditory cortex and visually-evoked gamma $(30-148 \mathrm{~Hz})$ oscillation amplitude

476 in the occipital lobe (Uhlhaas and Singer, 2011). Task-dependent changes involving higher-

477 order cognitive functions often saw effects that were differentiable between frontal and parietal 478 regions (Roux and Uhlhaas, 2014). The working memory load-dependent gamma power (50 - 
$100 \mathrm{~Hz}$ ) change reported by Kornblith et al. (2016) differed between task epochs and brain

480 regions. During and immediately after cue presentation, gamma power increased with higher

481 memory load in the parietal cortex but decreased with higher memory load in the prefrontal

482 cortex, while in the late delay period, there was no significant load-dependent gamma power

483 changes in either brain areas. Uhlhaas et al. (2009) reported age-dependent gamma (30 - 75

$484 \mathrm{~Hz}$ ) power changes in a face perception task for parietal electrodes only. Furthermore, the task

485 effect can be feature specific. Honkanen et al. (2015) saw load-dependent increases of gamma

$486(40-72 \mathrm{~Hz}$ and $80-120 \mathrm{~Hz})$ power for color and shape features but a decrease for location

487 features. At least some preliminary evidence exists for decreases in EEG gamma power in the

488 delay period of the Oculomotor Delayed Response task across adolescent development

489 (McKeon et al., 2020).

Oscillatory discharges increase early in postnatal development in non-human primates, as inferred by intracellular recording experiments in slice preparations (Gonzalez-Burgos et al.,

492 2015). It is possible therefore that gamma oscillations follow an inverted $U$ curve during

493 development. Gamma power has been also described in other animal models, e.g. revealing a

494 monotonic postnatal increase in prefrontal gamma power in some rodent studies (Bitzenhofer et

495 al., 2020). However, key differences have been discovered between animal models in the

496 developmental profile of excitatory-inhibitory circuits, including the lack of NMDA receptors on

497 adult frontal interneurons (Wang and Gao, 2009) as well as GABA synaptogenesis and

498 functional maturation being complete well before the onset of adolescence in the rodents (Le

499 Magueresse and Monyer, 2013).

Abnormally lower gamma power is commonly observed in schizophrenia (Woo et al.,

501 2010; Uhlhaas and Singer, 2013), generally attributed to a shift of the excitation/inhibition

502 balance towards a more excitable cortical state (Lisman, 2012). The decrease in gamma power

503 is specific for stimulus presentation and task engagement in patients with schizophrenia 
504 (Uhlhaas and Singer, 2010); spontaneous gamma power may be elevated in these patients, as

505 is during psychotic episodes and auditory hallucinations (Baldeweg et al., 1998; Spencer et al.,

506 2009; Grent-'t-Jong et al., 2018). In this context, our results would suggest that the primate

507 adolescence represents a state in the opposite end of the excitation/inhibition spectrum,

508 dominated by greater inhibition, which is again consistent with the idea of elevated inhibitory

509 drive in adolescence (Zhou et al., 2014). In turn, this result would suggest that schizophrenia

510 represents an aberrant, excessive decrease in inhibition, rather than a prolonged adolescent-

511 like state that failed to mature.

512 Through both empirical and modeling studies, a robust association has been established

513 between higher gamma power and recurrent activity. Indeed, we observed stronger gamma

514 power at recording sites with more neuronal information encoded. More subtly, the gamma

515 power and neuronal firing of local circuits became more aligned in the adult. Both findings agree

516 with the assumed role of gamma power at the microcircuit level. However, the overall direction

517 of change in gamma power between stages was inverted against that of neuronal firing and

518 behavior performance. These results suggest that during brain maturation, there might be

519 systematic changes at the molecular and/or network level prominent enough to mask changes

520 in features specific to a cognitive function of the brain region of interest. These effects would be

521 especially challenging to differentiate when only macroscopic measures of neural activity are

522 adopted. Bridging the processes underlying rhythmicity from the neuron to the circuit level will

523 be an important goal of future studies. 
REFERENCES

525

Anderson SA, Classey JD, Conde F, Lund JS, Lewis DA (1995) Synchronous development of pyramidal neuron dendritic spines and parvalbumin-immunoreactive chandelier neuron axon terminals in layer III of monkey prefrontal cortex. Neuroscience 67:7-22.

Baldeweg T, Spence S, Hirsch SR, Gruzelier J (1998) Gamma-band electroencephalographic oscillations in a patient with somatic hallucinations. Lancet 352:620-621.

Bitzenhofer SH, Pöpplau JA, Hanganu-Opatz I (2020) Gamma activity accelerates during prefrontal development. eLife 9:e56795.

Bourgeois JP, Goldman-Rakic PS, Rakic P (1994) Synaptogenesis in the prefrontal cortex of rhesus monkeys. Cereb Cortex 4:78-96.

Bunge SA, Dudukovic NM, Thomason ME, Vaidya CJ, Gabrieli JD (2002) Immature frontal lobe contributions to cognitive control in children: evidence from fMRI. Neuron 33:301-311.

Burgund ED, Lugar HM, Miezin FM, Schlaggar BL, Petersen SE (2006) The development of sustained and transient neural activity. Neuroimage 29:812-821.

Buzsaki G, Wang XJ (2012) Mechanisms of gamma oscillations. Annu Rev Neurosci 35:203225.

Compte A, Brunel N, Goldman-Rakic PS, Wang XJ (2000) Synaptic mechanisms and network dynamics underlying spatial working memory in a cortical network model. Cereb Cortex 10:910-923.

Compte A, Constantinidis C, Tegner J, Raghavachari S, Chafee MV, Goldman-Rakic PS, Wang XJ (2003) Temporally irregular mnemonic persistent activity in prefrontal neurons of monkeys during a delayed response task. J Neurophysiol 28:3441-3454.

Constantinidis C, Luna B (2019) Neural Substrates of Inhibitory Control Maturation in Adolescence. Trends Neurosci 42:604-616.

Davidson MC, Amso D, Anderson LC, Diamond A (2006) Development of cognitive control and executive functions from 4 to 13 years: evidence from manipulations of memory, inhibition, and task switching. Neuropsychologia 44:2037-2078.

Dienel SJ, Lewis DA (2019) Alterations in cortical interneurons and cognitive function in schizophrenia. Neurobiol Dis 131:104208.

Fries P (2009) Neuronal Gamma-Band Synchronization as a Fundamental Process in Cortical Computation. Annual Review of Neuroscience 32:209-224.

Fry AF, Hale S (2000) Relationships among processing speed, working memory, and fluid intelligence in children. Biol Psychol 54:1-34.

Gathercole SE, Pickering SJ, Ambridge B, Wearing H (2004) The structure of working memory from 4 to 15 years of age. Dev Psychol 40:177-190.

Giedd JN, Rapoport JL (2010) Structural MRI of Pediatric Brain Development: What Have We Learned and Where Are We Going? Neuron 67:728-734.

Goldman-Rakic PS (1994) Working memory dysfunction in schizophrenia. J Neuropsychiatry Clin Neurosci 6:348-357.

Gonzalez-Burgos G, Miyamae T, Pafundo DE, Yoshino H, Rotaru DC, Hoftman G, Datta D, Zhang Y, Hammond M, Sampson AR, Fish KN, Ermentrout GB, Lewis DA (2015) Functional Maturation of GABA Synapses During Postnatal Development of the Monkey Dorsolateral Prefrontal Cortex. Cereb Cortex 25:4076-4093.

Grent-'t-Jong T, Gross J, Goense J, Wibral M, Gajwani R, Gumley Al, Lawrie SM, Schwannauer M, Schultze-Lutter F, Navarro Schroder T, Koethe D, Leweke FM, Singer W, Uhlhaas PJ (2018) Resting-state gamma-band power alterations in schizophrenia reveal E/I-balance abnormalities across illness-stages. Elife 7.

Hoftman GD, Lewis DA (2011) Postnatal developmental trajectories of neural circuits in the primate prefrontal cortex: identifying sensitive periods for vulnerability to schizophrenia. Schizophr Bull 37:493-503. 
574

575

576

577

578

579

580

581

582

583

584

585

586

587

588

589

590

591

592

593

594

595

596

597

598

599

600

601

602

603

604

605

606

607

608

609

610

611

612

613

614

615

616

617

618

619

620

621

622

Honkanen R, Rouhinen S, Wang SH, Palva JM, Palva S (2015) Gamma Oscillations Underlie the Maintenance of Feature-Specific Information and the Contents of Visual Working Memory. Cerebral Cortex 25:3788-3801.

Howard MW, Rizzuto DS, Caplan JB, Madsen JR, Lisman J, Aschenbrenner-Scheibe R, Schulze-Bonhage A, Kahana MJ (2003) Gamma Oscillations Correlate with Working Memory Load in Humans. Cereb Cortex 13:1369-1374.

Huttenlocher PR, Dabholkar AS (1997) Regional differences in synaptogenesis in human cerebral cortex. The Journal of comparative neurology 387.

Jensen O, Kaiser J, Lachaux JP (2007) Human gamma-frequency oscillations associated with attention and memory. Trends Neurosci 30:317-324.

Kajikawa Y, Schroeder Charles E (2011) How Local Is the Local Field Potential? Neuron 72:847-858.

Klingberg T, Forssberg $\mathrm{H}$, Westerberg $\mathrm{H}$ (2002) Training of working memory in children with ADHD. J Clin Exp Neuropsychol 24:781-791.

Kornblith S, Buschman TJ, Miller E (2016) Stimulus Load and Oscillatory Activity in Higher Cortex. Cerebral cortex.

Kwon H, Reiss AL, Menon V (2002) Neural basis of protracted developmental changes in visuospatial working memory. Proc Natl Acad Sci U S A 99:13336-13341.

Le Magueresse C, Monyer H (2013) GABAergic interneurons shape the functional maturation of the cortex. Neuron 77:388-405.

Lewis DA, Cruz D, Eggan S, Erickson S (2004) Postnatal development of prefrontal inhibitory circuits and the pathophysiology of cognitive dysfunction in schizophrenia. Annals of the New York Academy of Sciences 1021:64-76.

Li S, Zhou X, Constantinidis C, Qi XL (2020) Plasticity of Persistent Activity and Its Constraints. Front Neural Circuits 14:15.

Lisman J (2012) Excitation, inhibition, local oscillations, or large-scale loops: what causes the symptoms of schizophrenia? Curr Opin Neurobiol 22:537-544.

Luna B, Thulborn KR, Munoz DP, Merriam EP, Garver KE, Minshew NJ, Keshavan MS, Genovese CR, Eddy WF, Sweeney JA (2001) Maturation of widely distributed brain function subserves cognitive development. Neuroimage 13:786-793.

Lundqvist M, Herman P, Warden MR, Brincat SL, Miller EK (2018) Gamma and beta bursts during working memory readout suggest roles in its volitional control. Nat Commun 9:394.

Lundqvist M, Rose J, Herman P, Brincat SL, Buschman TJ, Miller EK (2016) Gamma and Beta Bursts Underlie Working Memory. Neuron 90:152-164.

McKeon S, Calabro F, Luna B (2020) Development of EEG-derived spectral processing of working memory through adolescence In: Flux Congress, pp 82-82.

Olesen PJ, Nagy Z, Westerberg H, Klingberg T (2003) Combined analysis of DTI and fMRI data reveals a joint maturation of white and grey matter in a fronto-parietal network. Brain Res Cogn Brain Res 18:48-57.

Olesen PJ, Macoveanu J, Tegner J, Klingberg T (2007) Brain activity related to working memory and distraction in children and adults. Cereb Cortex 17:1047-1054.

Pesaran B, Pezaris JS, Sahani M, Mitra PP, Andersen RA (2002) Temporal structure in neuronal activity during working memory in macaque parietal cortex. Nat Neurosci 5:805-811.

Ray S, Maunsell JH (2011) Different origins of gamma rhythm and high-gamma activity in macaque visual cortex. PLoS Biol 9:e1000610.

Roux F, Uhlhaas PJ (2014) Working memory and neural oscillations: alpha-gamma versus theta-gamma codes for distinct WM information? Trends Cogn Sci 18:16-25. 

cortex gamma synchronization and auditory hallucination symptoms in schizophrenia. BMC Neurosci 10:85.

Uhlhaas PJ, Singer W (2010) Abnormal neural oscillations and synchrony in schizophrenia. Nat Rev Neurosci 11:100-113.

Uhlhaas PJ, Singer W (2011) The Development of Neural Synchrony and Large-Scale Cortical Networks During Adolescence: Relevance for the Pathophysiology of Schizophrenia and Neurodevelopmental Hypothesis. Schizophrenia Bulletin 37:514-523.

Uhlhaas PJ, Singer W (2013) High-frequency oscillations and the neurobiology of schizophrenia. Dialogues Clin Neurosci 15:301-313.

Uhlhaas PJ, Roux F, Rodriguez E, Rotarska-Jagiela A, Singer W (2010) Neural synchrony and the development of cortical networks. Trends Cogn Sci 14:72-80.

Uhlhaas PJ, Roux F, Singer W, Haenschel C, Sireteanu R, Rodriguez E (2009) The development of neural synchrony reflects late maturation and restructuring of functional networks in humans. Proc Natl Acad Sci U S A 106:9866-9871.

Ullman H, Almeida R, Klingberg T (2014) Structural maturation and brain activity predict future working memory capacity during childhood development. J Neurosci 34:1592-1598.

Wang H-X, Gao W-J (2009) Cell Type-Specific Development of NMDA Receptors in the Interneurons of Rat Prefrontal Cortex. Neuropsychopharmacology 34:2028-2040.

Wang X-J (2010) Neurophysiological and Computational Principles of Cortical Rhythms in Cognition. Physiological Reviews 90:1195-1268.

Woo T-UW, Spencer K, McCarley RW (2010) Gamma Oscillation Deficits and the Onset and Early Progression of Schizophrenia. Harvard Review of Psychiatry 18:173-189.

Zhou X, Qi XL, Constantinidis C (2016a) Distinct Roles of the Prefrontal and Posterior Parietal Cortices in Response Inhibition. Cell Rep 14:2765-2773.

Zhou X, Zhu D, Qi XL, Lees CJ, Bennett AJ, Salinas E, Stanford TR, Constantinidis C (2013) Working Memory Performance and Neural Activity in the Prefrontal Cortex of Peripubertal Monkeys. J Neurophysiol 110:2648-2660.

Zhou X, Zhu D, King SG, Lees CJ, Bennett AJ, Salinas E, Stanford TR, Constantinidis C (2016b) Behavioral response inhibition and maturation of goal representation in prefrontal cortex after puberty. Proc Natl Acad Sci U S A 113:3353-3358.

Zhou X, Zhu D, Qi XL, Li S, King SG, Salinas E, Stanford TR, Constantinidis C (2016c) Neural correlates of working memory development in adolescent primates. Nat Commun 7:13423.

Zhou X, Zhu D, Katsuki F, Qi XL, Lees CJ, Bennett AJ, Salinas E, Stanford TR, Constantinidis C (2014) Age-dependent changes in prefrontal intrinsic connectivity. Proc Natl Acad Sci U S A 111:3853-3858. 


\section{Figure Legends}

A

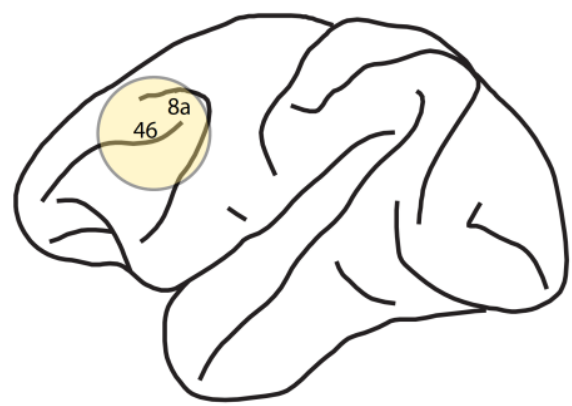

B

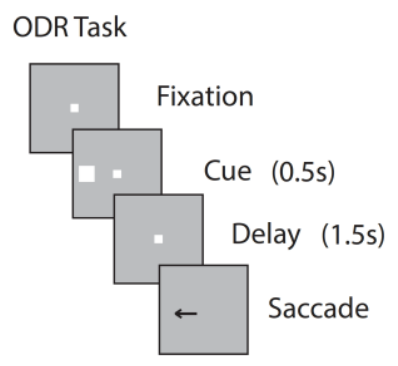

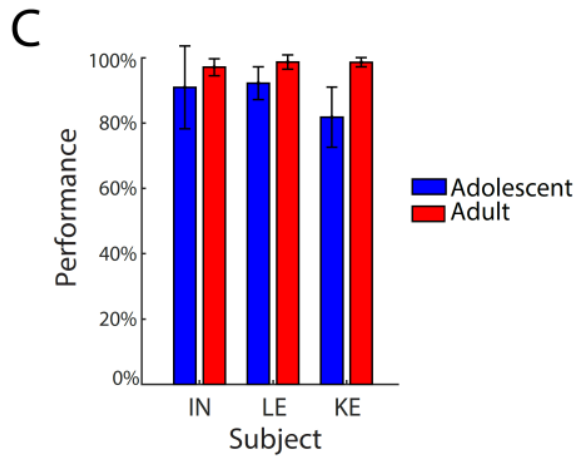

663
Figure 1. Brain regions recorded, task design and behavior performance. A: Schematic diagram of the monkey brain with locations of neurophysiological recordings in the dorsolateral prefrontal cortex indicated (areas 8a and 46). B: The oculomotor delayed response task required the subject to saccade to a remembered location after a 1.5 second delay. C: Behavior performance of the three subjects used in this study in two stages 1.6-2.1 years apart, respectively referred to as the "adolescent" and "adult" stage. Mean percentage of correct trials in ODR task, excluding breaks in fixation, for young: 88\%; adult: 98\%. Error bars represent standard deviation. 

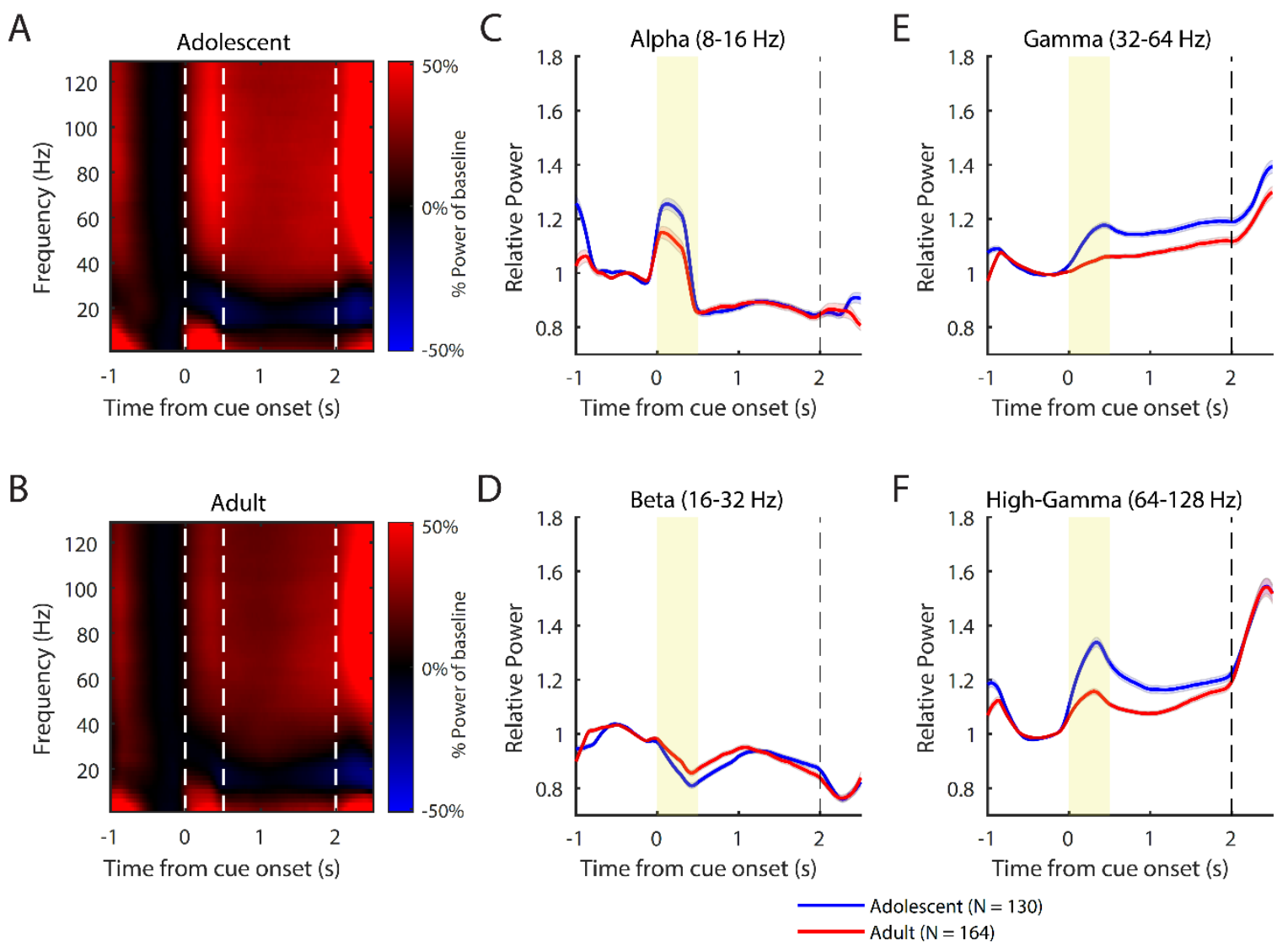

G

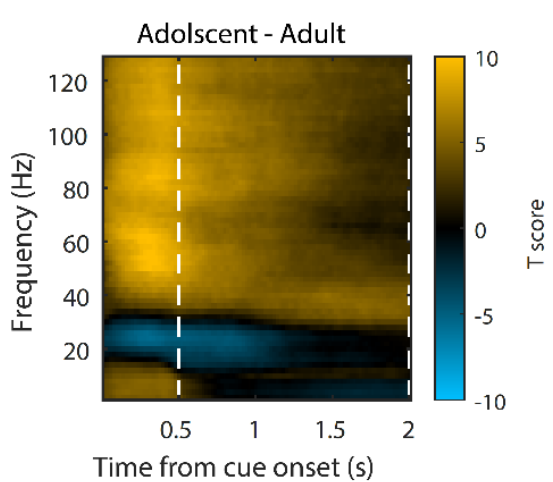

$\mathrm{H}$

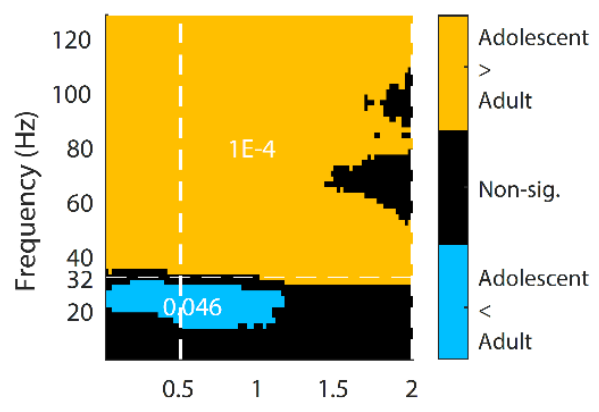

Time from stim. onset (s)
673

674

675

676

677

678

679

680

681

682

683

Figure 2. dIPFC LFP power changes during adolescent development. $A$, B: Population average across electrodes and sessions of moment-by-moment LFP spectral power in proportion to that in the last 500 milliseconds prior to cue onset for LFP recording sites at the adolescent $(\mathrm{N}=130)$ and adult $(\mathrm{N}=164)$ stage. $\mathrm{C}-\mathrm{F}$ : Population averages of LFP power evolutions aligned to cue onset in the frequency bands between 8-16, 16-32, 32-64 and 64-128 $\mathrm{Hz}$ at sites recorded in the adolescent $(\mathrm{N}=130)$ and adult $(\mathrm{N}=164)$ stage. $\mathrm{G}$ : Uncorrected 2sample t-test $t$ values comparing the moment-by-moment LFP spectral power in proportion to that in the last 500 milliseconds prior to cue onset between the adolescent $(N=130)$ and adult $(\mathrm{N}=164)$ stage. Positive values correspond to larger adolescent power. $\mathrm{H}$ : Significant clusters found in the permutation test based on $t$ values in $A$. The number in each cluster represents the bootstrapped $p$ value. 
A

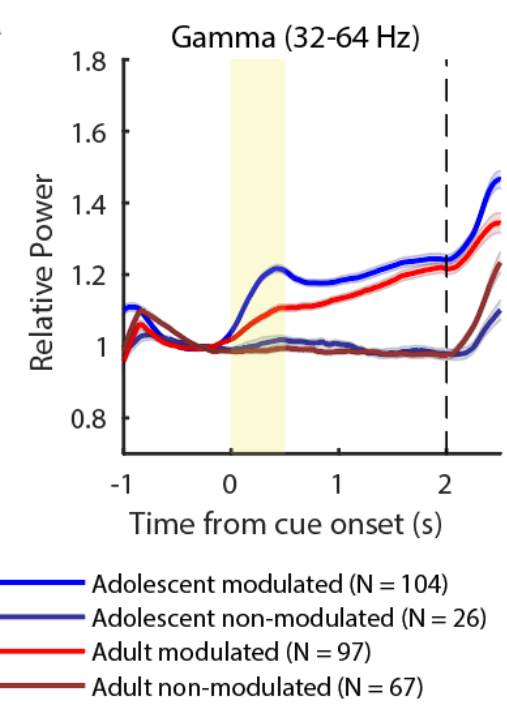

B

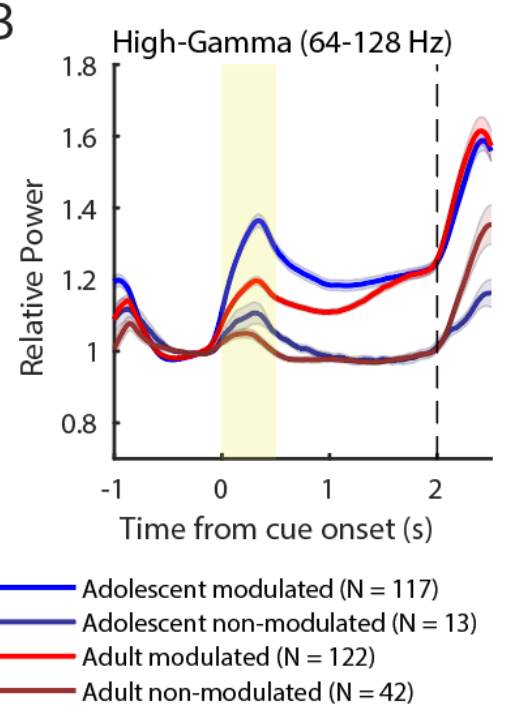

685 Figure 3. Heterogeneity in gamma and high-gamma LFP power changes across recording 686 sites. A, B: LFP band power evolutions of sites grouped according to the level of changes

687 (significant/non-significant) in gamma (adolescent 104/26, adult 97/67) or high-gamma

688 (adolescent 117/13, adult 122/42) power respectively during the working memory delay. Shaded 689 areas represent SEM. 
A

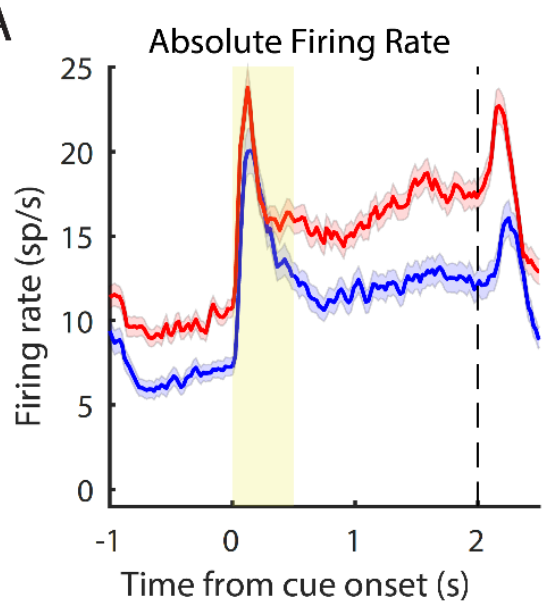

B

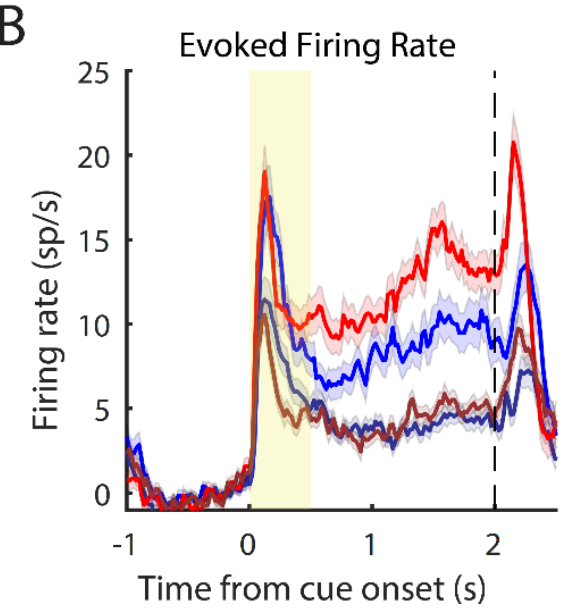

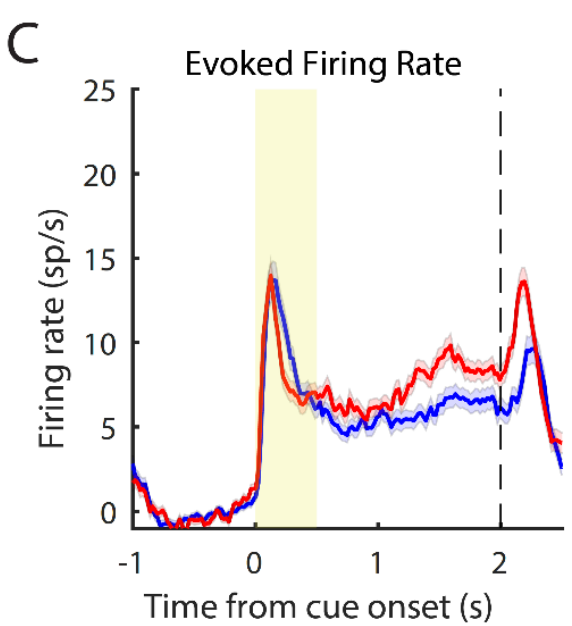

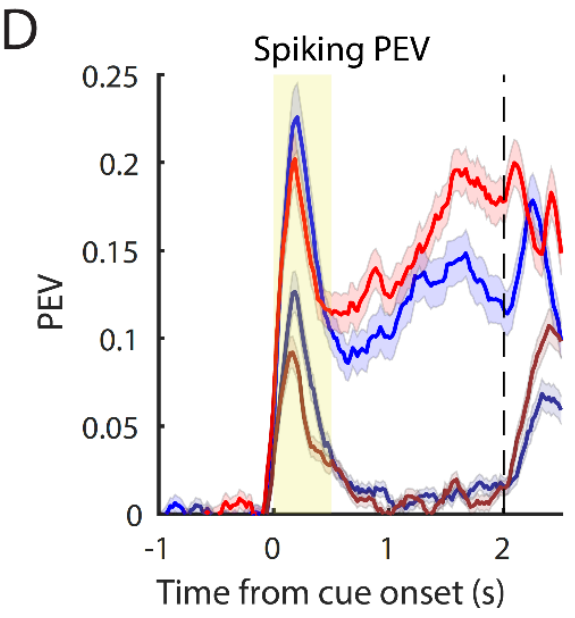

Figure 4. dIPFC neuronal spiking and information encoding in the ODR task. A, C: Population averages of neuronal raw firing rate and evoked firing rate by subtracting the average in the 1-second fixation period for neurons recorded in the adolescent $(\mathrm{N}=298)$ and adult $(\mathrm{N}=392)$ stages. $\mathrm{B}, \mathrm{D}$ : Average evoked firing rate and proportion of its explained variance (PEV) by cue location after dividing the neurons according to differential delay period firing (selective/non-selective) in the adolescent (116/182) and adult (157/235) stage. 
A

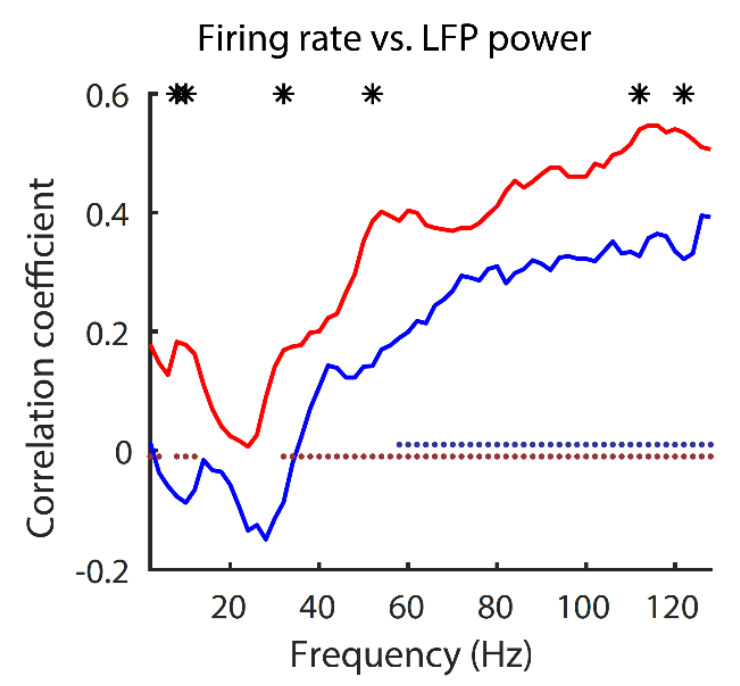

C

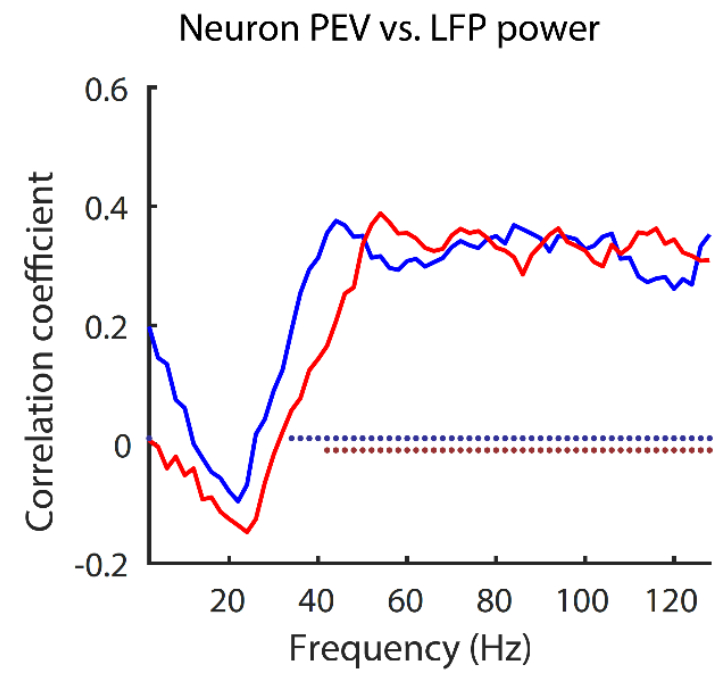

B

Firing rate vs. LFP power (reduced set)

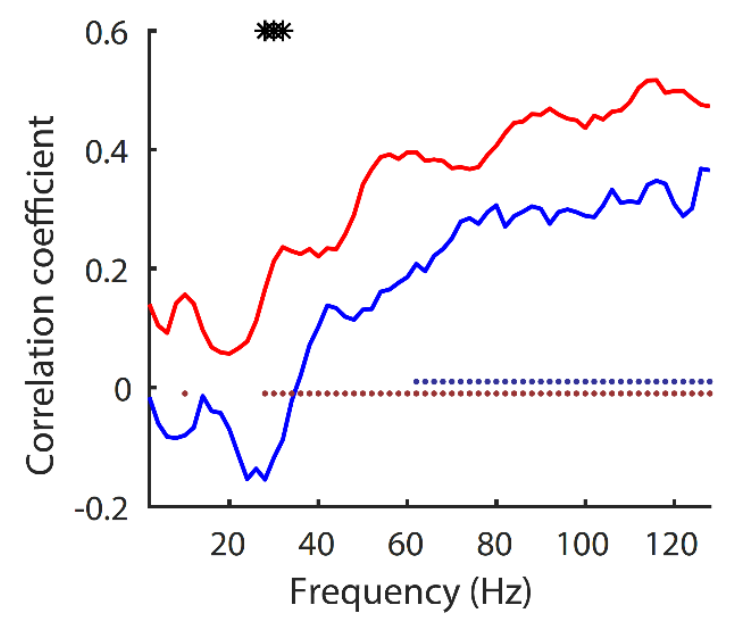

D

Neuron PEV vs. LFP power (reduced set)

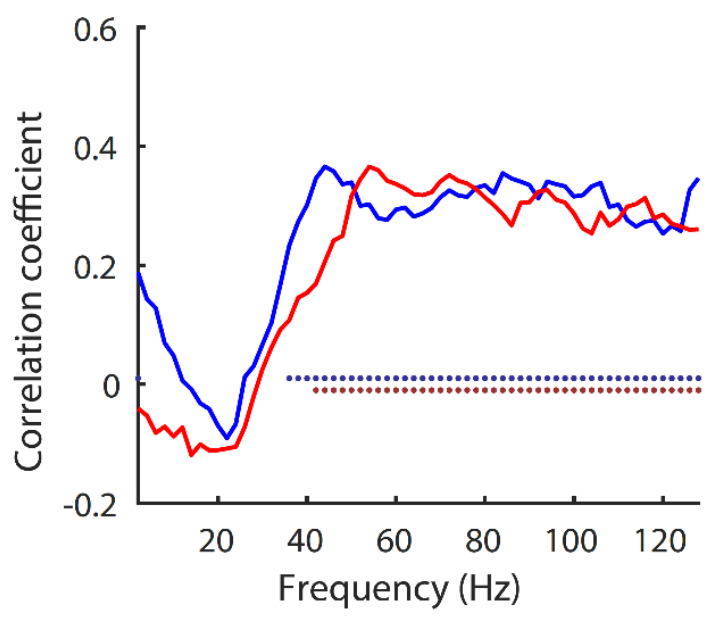

Figure 5. Neuron-by-neuron correlations between spiking activity and LFP power. A: Spearman's correlation coefficient between neuronal firing rate and LFP power for signals recorded from the same electrode. C: Spearman's correlation coefficient between neuronal PEV and LFP power for signals recorded from the same electrode. B and D: Same as A and C but for a firing rate-matched subset of neurons. Solid dots indicate significant correlations (Bonferroni-Holm-corrected type I error rate $<0.05$ ). Asterisks stand for significant differences between the adolescent and the adult (Fisher's Z transform, Bonferroni-Holm-corrected type I error rate $<0.05$ ). 

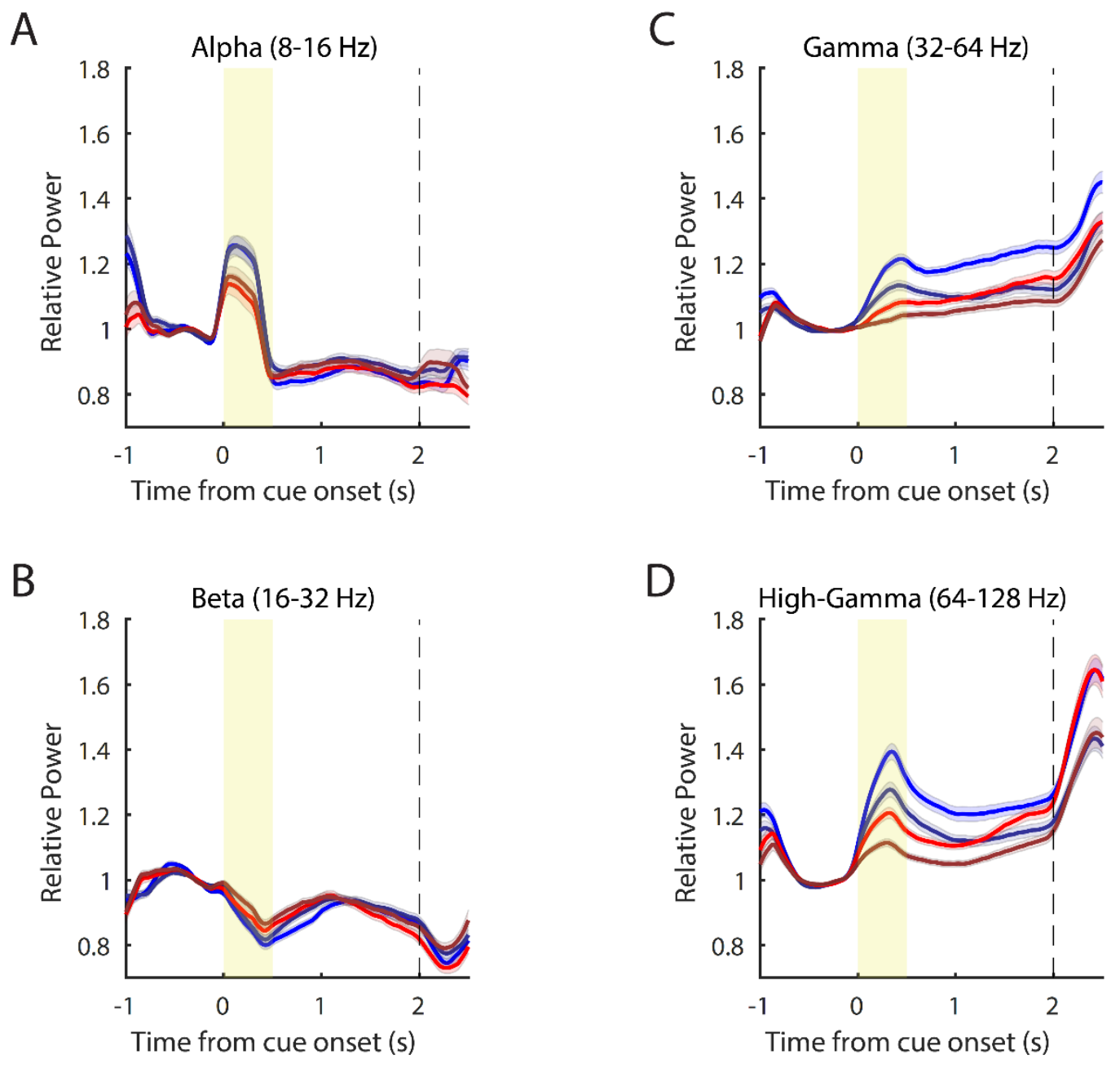

Adolescent with selective neurons $(\mathrm{N}=69)$

Adolescent without selective neurons $(\mathrm{N}=61)$

Adult with selective neurons $(\mathrm{N}=77)$

Adult without selective neurons $(\mathrm{N}=87)$

Figure 6. LPF power modulation as a function neuronal information encoding. LFP band power evolutions of sites grouped according to whether spatially-selective neurons were recorded at the corresponding sites in the adolescent (69/61) and adult (77/87) stage. 

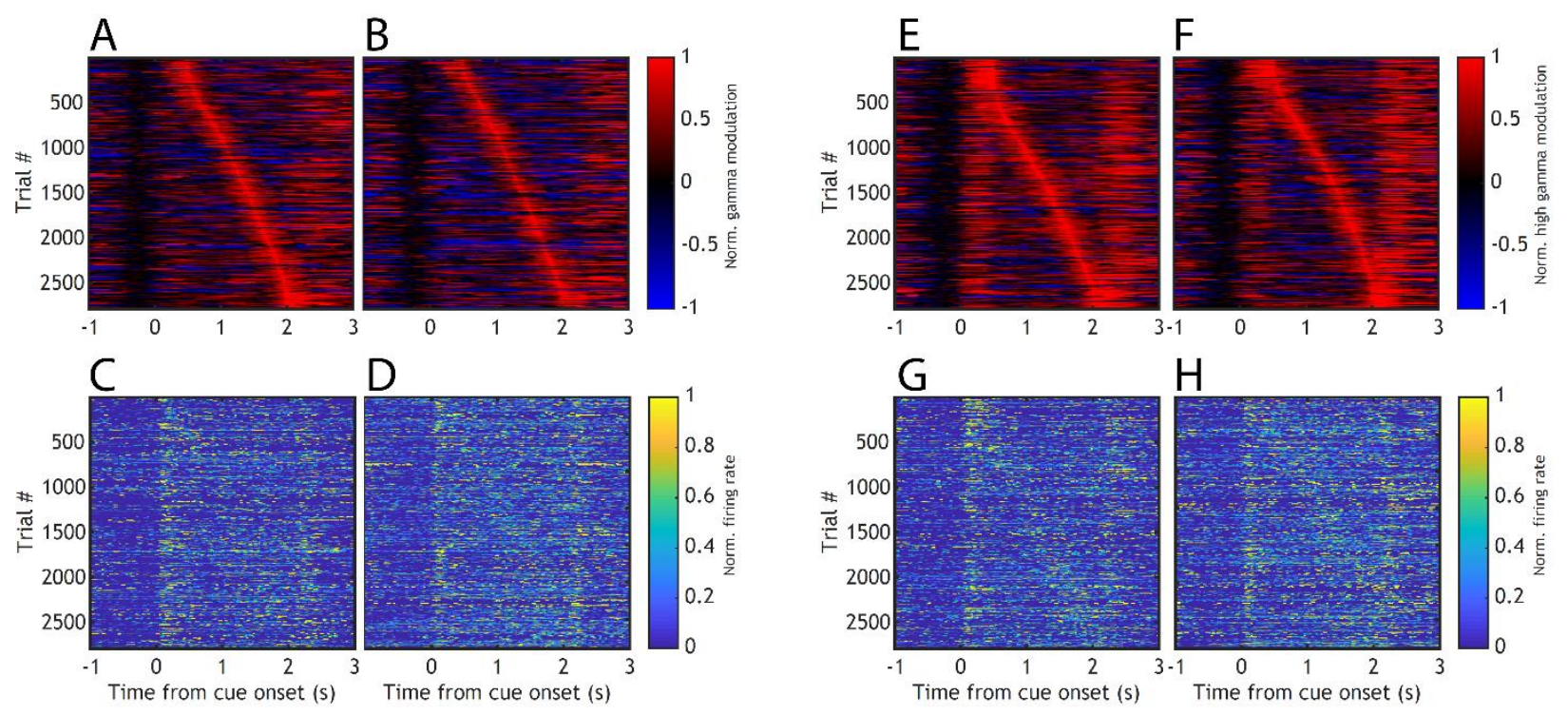

711 Figure 7. Trial-by-trial temporal evolution of neuronal firing and matched LFP power. A:

712 Adolescent single-trial gamma band power arranged according to peak timing in the delay

713 period $(\mathrm{N}=2792)$. $\mathrm{B}$ : Same as $\mathrm{A}$ for the adult LFP $(\mathrm{N}=3393)$. $\mathrm{C}$, $\mathrm{D}$ : Normalized neuronal firing

714 rate (convolved with a 50-millisecond boxcar kernel) corresponding to the same trials in $A$ and

715 B. E-H: Same as A-D for high gamma power. 


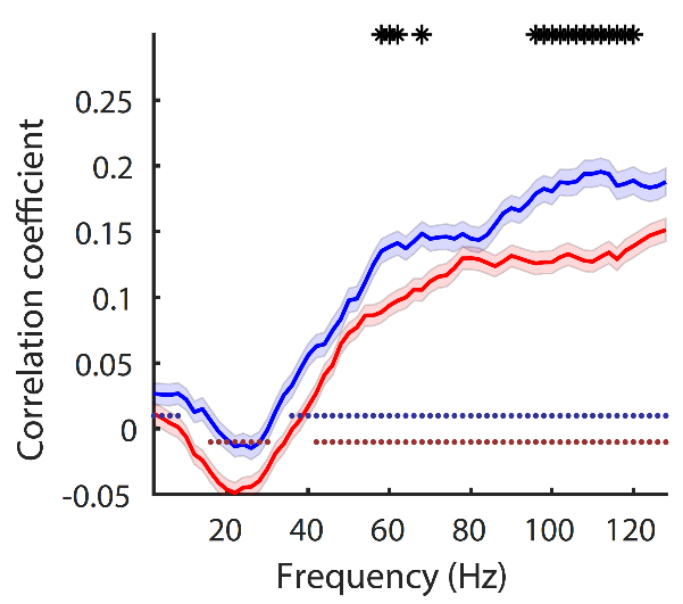

717 Figure 8. Average temporal correlation of LFP power with neuronal spiking. Solid lines are 718 average Spearman's correlation coefficient for the adolescent and adult stages respectively.

719 Shaded area represent SEM. Solid dots indicate average correlations significantly different from 720 zero (signed-rank test, Bonferroni-Holm-corrected type I error rate $<0.05$ ). Asterisks stand for 721 significant differences between the adolescent and the adult (Wilcoxon's rank-sum test, 722 Bonferroni-Holm-corrected type I error rate < 0.05). 